\title{
NOCARDIOSIS IN A PATIENT WITH LUPUS NEPHRITIS
}

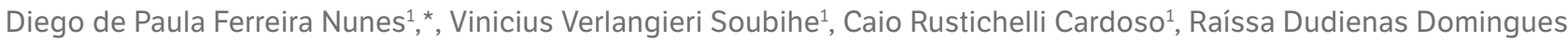
Pereira $^{1}$, Simone Appenzeller ${ }^{1}$, Lilian Tereza Lavras Costallat ${ }^{1}$

1.Universidade Estadual de Campinas, Campinas (SP), Brazil.

*Corresponding author: diegopfn@gmail.com

\section{BACKGROUND}

Acute confusional state in systemic lupus erythematosus (SLE) is always a challenge in the assessment. Infectious, metabolic, and neurological causes must be ruled out before associating disease activity. Infection is the leading cause of mortality in patients with systemic lupus erythematosus. Factors such as disease activity, immunosuppression and ethnicity are important factors that contribute to this scenario.

\section{CASE REPORT}

A 37-year-old women diagnosed with SLE (ANA +, anti-DNA reagent, arthritis, nephritis with class IV biopsy, photosensitivity) since 2010, previously presented with three episodes of nephritis with poor adherence to treatment, with a new renal flare in 2020 after discontinuation of maintenance phase treatment. In the third month of cyclophosphamide, the patient started to have a dry cough, evolving with nodular skin lesions on the back, chest and limbs. She chose to suspend the infusion of cyclophosphamide for investigation. She progressed with acute confusional state and was admitted to emergency. Skull tomography showing expansive lesions centered in the left temporal lobe, with contrast-enhanced ring parietal enhancement with midline deviation (suggestive of brain abscess), chest tomography with elongated opacity located in the superior lingular segment and left hilar region. Drainage of the brain abscess improved consciousness. Culture of brain and skin abscess were positive for Nocardia farcinica infection. Treatment was done with 6 weeks of intravenous imipenem and sulfamethoxazole trimethoprim, followed by oral therapy with sulfamethoxazole trimethoprim for 1 year. She improvement significantly during treatment, after intravenous therapy opted to start mycophenolate $3 \mathrm{~g} /$ day for nephritis induction seen in the patient with renal activity, showing partial response after induction.

\section{CONCLUSION}

Infectious causes leading to altered level of consciousness should always be ruled out in lupus patients. Nocardiosis is an uncommon and potentially serious condition, with prolonged treatment. The case described shows lupus nephritis with impossibility of immunosuppressive treatment due to the current infection. Presenting good outcome after proper treatment.

\section{KEYWORDS}

Systemic lupus erythematosus, Nocardiosis, Lupus nephritis. 